\title{
Thrombolytic therapy
}

\author{
P. T. FLUTE \\ From the Department of Haematology, King's College Hospital Medical School, London
}

Enzymatic attack on preformed thrombi is a very attractive prospect. Many reports have followed that from Tillett, Johnson, and McCarty (1955) who were the first to try and dissolve thrombi within the vascular system in man. This was in 1955; they used streptokinase some 22 years after its first isolation. Now, eight years later, urokinase, another activator, is available in a form suitable for intravascular use; but there is still no general agreement either on how these enzymes should be used, nor, though isolated successes have been reported, whether they can be of real clinical value. Not only must this treatment dissolve thrombi but it must do so in time to produce a satisfactory clinical result. If an artery is obstructed only a few hours are available in which to restore blood flow before tissues will die. Dosage must therefore be carefully controlled in order to achieve the best result in the shortest possible time. Too little may be ineffective, too much enzyme is undoubtedly dangerous. I should like to discuss how the best dosage can be decided for each individual patient.

No discussion of enzyme therapy would be complete without a reference to the extensive pioneer work of Sherry and his associates in St. Louis (Sherry, Fletcher, and Alkjaersig, 1959). It is Sherry's hypothesis concerning the mechanism of thrombolysis which has formed the basis of almost all the subsequent work on this subject. He suggests that the breakdown of a clot depends more on the concentration of plasminogen activator which can gain access to it than on the concentration of plasmin to which it is exposed. Activators such as streptokinase or urokinase convert plasminogen, a normal circulating globulin, to plasmin. Plasmin formed from the plasminogen within the thrombus attacks fibrin, splitting it into soluble polypeptides. Plasmin formed from circulating plasminogen may attack many other proteins. Fortunately combination between plasmin and the antiplasmins of the blood usually prevents this undesirable event but even with adequate levels of antiplasmins fibrinogen may to some extent be digested. Both the actual lack of fibrinogen and the presence of fibrinogen breakdown products, which interfere with the poly- merization of fibrin, contribute to the bleeding state which is the worst danger of this type of therapy.

This then is the basis on which my colleagues and I at King's College Hospital have chosen to work. We have conducted a series of experiments in some 50 dogs and have treated a small series of patients. While I will leave a discussion of the clinical results to my colleague, Mr. Tsapogas, I should perhaps say at the outset that we have no doubt that both streptokinase and urokinase will dissolve thrombi in the arteries of both animals and man.

I would like first to describe some experiments which can be performed in the laboratory. When activator is added to plasma in the test tube and measurements made of the resultant fibrinolytic activity and of changes in fibrinogen, plasminogen, and blood-clotting factors after $\mathbf{3 0}$ minutes' incubation, different effects are seen, depending on the amount of activator added. Moreover, an amount of activator which will produce profound effects in one individual will fail to produce any change in another.

Fibrinolytic activity may be tested on a standard bovine fibrin plate. A clot is formed as a sheet on the bottom of a petri dish and three drops of plasma applied to the surface of the clot. The area of lysis produced after 20 hours' incubation at $37^{\circ} \mathrm{C}$. is proportional to the fibrinolytic activity of the plasma.

Normal human plasma has no fibrinolytic activity on standard bovine fibrin plates. Small additions of activator, either streptokinase or urokinase, to plasma fail to confer any fibrinolytic activity on that plasma, although the same amounts of activator dissolved in buffer produce large areas of lysis on the same plates. It is not until a certain critical amount of activator is exceeded that fibrinolytic activity first appears in the plasma, and the amount of activator required to produce this effect varies widely in different subjects, and even to different activators in the same individual. In this model system the fibrin plate may be taken to represent a thrombus. If the whole plasma fails to produce lysis of the plate, which has a simple structure, virtual absence of inhibitors, and adequate plasminogen, surely it is unreasonable to expect 
thrombi to be dissolved in the body by the effects of that plasma impinging upon them.

The amount of activator which must be added to the plasma of any individual in order to confer fibrinolytic activity on the whole plasma when it is tested on a fibrin plate therefore assumes considerable importance in relation to thrombolytic therapy. It will be called the critical dose, and is measured in activator units per millilitre of plasma.

The nature of the inhibition of activator at levels below the critical dose is still uncertain. It may be that the effect is due to the presence of a true anti-activator; while this is certainly the case for anti-streptokinase antibody, specific inhibitors against urokinase have yet to be clearly demonstrated. Antiplasmins play some part in this inhibition because their destruction by acidification of the plasma or the isolation of the euglobulin fraction of the plasma, which does not contain antiplasmins, allows fibrinolytic activity to be demonstrated on the fibrin plate even with amounts of activator which fail to produce activity in untreated plasma. Large amounts of antiplasmins are still present even when the critical dose of activator is exceeded by very large amounts. This can be shown by direct measurement and by the fact that acidification of plasma, which destroys antiplasmins, still increases the area of lysis obtained on a fibrin plate beyond that given by the non-acidified plasma. Nevertheless, only traces of free plasmin can be demonstrated and the amount does not rise progressively as the activator level is increased.

Measurements of plasminogen, fibrinogen, and clotting factors in plasma to which variable amounts of activator have been added show no change in these factors until the critical dose of activator is exceeded. With additions of activator in excess of the critical dose there is a fall, which is directly proportional to the amount of activator added, in all these factors.

When a patient is to be treated there is no time to test the effect of additions of activator to his plasma on a fibrin plate. There is, however, a simple way of measuring the critical dose. This is by observing the lysis times of plasma clots incubated at $37^{\circ} \mathrm{C}$. containing increasing amounts of activator. A suitable system is $0 \cdot 1 \mathrm{ml}$. volumes of bovine thrombin, activator dilution, and citrated plasma, added in that order to a glass tube at $37^{\circ} \mathrm{C}$. As shown by Tillett et al. (1955), the reciprocal of the lysis time plotted against the logarithm of the activator concentration gives a straight line. However, the relationship is strictly linear only for certain definite proportions of activator and plasma, particularly those giving lysis times between two and 20 minutes. If the line is prolonged to infinite lysis time this point

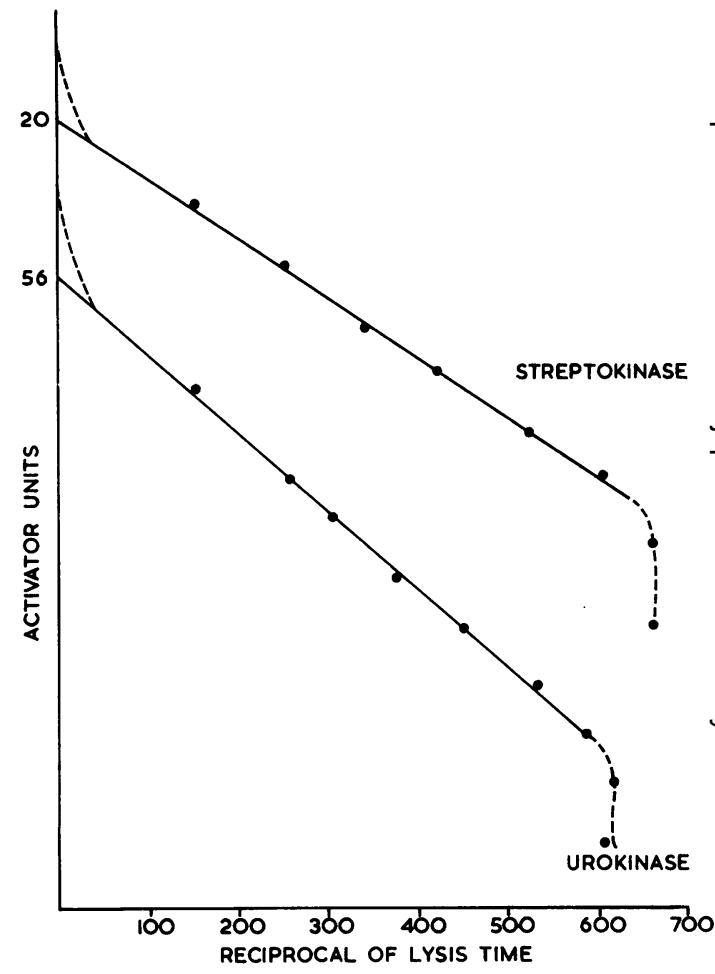

FIG. 1. The determination of the critical dose.

may be used to indicate the critical dose. Othe? workers have arbitrarily taken the intercepts on this line at $\mathbf{2 0}$ minutes or $\mathbf{1 0}$ minutes as an index of the effective dose; this will make only slight differences to the calculated amount.

Figure 1 shows such a graph for streptokinase anc urokinase in the same individual. For most of these points the relationship is linear. As the amount of activator increases, a plateau is reached, beyon of which the lysis time does not decrease further. The position of this plateau is dependent upon the amount of plasminogen in the plasma. At the lower end of the scale, small doses of activator stilp shorten the lysis time, but there is no longer the same linear relationship.

Of those who have used this method, some have added the activator to plasma, some to whole blood and some to E.D.T.A.-treated blood. It can be shown that as regards the linear portion of the line the lysis time is not affected by dilution, the presence्ष of calcium ions, E.D.T.A., or platelets. When? small amounts of activator are present and the lysis times are longer than 20 minutes each of these factors then has a profound effect. 
In one individual the dose was measured as follows:-

\begin{tabular}{|c|c|c|}
\hline Lysis Times (min.) & \multicolumn{2}{|c|}{ Addition of Activator (Units/ml.) } \\
\hline & Streptokinase & Urokinase \\
\hline $\begin{array}{r}\text { Infinite } \\
20 \\
10\end{array}$ & $\begin{array}{l}21 \\
24 \\
31\end{array}$ & $\begin{array}{l}58 \\
72 \\
92\end{array}$ \\
\hline
\end{tabular}

Of the greatest importance is that each of these results is very close to what has so far been called the critical dose, that is, they represent the smallest amount of activator which, when added to plasma, will confer fibrinolytic activity on that plasma when it is tested on a fibrin plate. If the plate is attacked, it is at least possible that a thrombus might be attacked also. though doses below this amount would certainly fail. Measurements of the critical dose of streptokinase in 77 subjects show a mean value of 53 units $/ \mathrm{ml}$. with a range of 13 to 170 units $/ \mathrm{ml}$. of plasma.

Until the question of the nature of the inhibition is resolved, I prefer to think of this as the critical dose, rather than as the thrombolysis-inducing dose, which may be overoptimistic, or even than the fibrinolysis-inducing dose, which again is not strictly accurate because the lysis time is shortened at doses below this.

Once the critical dose of activator has been determined, this knowledge may be used in two ways. The activator may be dissolved in saline so that $1 \mathrm{ml}$. contains the critical dose in units $/ \mathrm{ml}$. This solution is then infused at the rate of 1 to $2 \mathrm{ml}$. per minute directly into the blocked vessel. A level of activator equivalent to the critical dose is thus produced in the region of the clot, but not elsewhere in the body, owing to dilution by the circulating blood.

Alternatively, the critical dose in units per millilitre is multiplied by the plasma volume in millilitres and the total dose given intravenously over 15 minutes; this should produce activity throughout the vascular system. If the dose has been correctly calculated, there should be demonstrable fibrinolytic activity if the fresh plasma is placed on a fibrin plate, together with a fall in blood plasminogen and fibrinogen levels. The thrombin clotting time is likely to be prolonged and breakdown products interfering with fibrin polymerization may be demonstrated if serum, from which the clot has been removed, is $25 \%$ saturated with ammonium sulphate. Normal serum gives no precipitate with this treatment, but if fibrinogen or perhaps some of its breakdown products are still present due to inter- ference with fibrin polymerization a precipitate occurs.

If the critical dose is exceeded all these changes are accentuated. Continued infusions also produce a progressive fall in plasminogen and fibrinogen but the values appear to stabilize after four or five hours at levels dependent on the dose administered. If pushed to the extreme, bleeding is likely, even though antiplasmins can still be demonstrated in the blood.

Once the initial dose has produced fibrinolytic activity the level of activator has to be maintained. The dose to be given by continuous infusion will depend upon the rate of clearance of activator from the circulation. In the case of streptokinase there will also be a variable amount of antibody which, once neutralized, will play no further part so that the continuing infusion will not need to maintain the whole of the original activator level. In the case of urokinase no inhibitor appears to be finally neutralized. If fibrinolytic activity is produced in a dog by a single injection of activator the effects usually last for less than one hour whether the activator is urokinase or streptokinase. We have therefore given continuous infusions of urokinase containing the original dose every one to two hours. Nevertheless, once the dose has been calculated it is certainly necessary to make hourly measurements of activity, plasminogen, and fibrinogen to ensure that the requisite levels of activator are achieved but not exceeded.

Finally, if this type of therapy is to be correctly assessed it seems pointless to go to all this trouble unless an objective measure of clinical effectiveness is also available.

\section{REFERENCES}

Sherry, S., Fletcher, A. P., and Alkjaersig, N. (1959). Physiol. Rev., 39, 343.

Tillett, W. S., Johnson, A. J., and McCarty, W. R. (1955). J. clin. Invest., 34, 169.

MR. TSAPOGAS said that if thrombosis was sufficiently extensive the time taken to dissolve the thrombus in the peripheral vessels by thrombolytic agents might be so long that the limb died. It is consequently now the policy at King's College Hospital in cases of extensive thrombosis to disobliterate the main artery by thrombectomy and, if arteriography confirms that the distal vessels are still blocked, to follow this up with fibrinolytic therapy.

DR. CROSBIE stated that in Liverpool they would soon have a high pressure oxygen chamber, which, it was hoped, would preserve the life of a thrombosed limb for a period sufficiently long to permit controlled fibrinolytic therapy. 